\title{
Apreciaciones sobre la funcionalidad familiar en personas desmovilizadas de grupos armados insurgentes asentadas en el Eje Cafetero
}

\author{
Melany Reina Mosquera'; Dahiana Giraldo Ríos²; José Alonso Andrade Salazar³
}

RESUMEN Esta es una investigación cualitativa, de tipo exploratoria, cuyo objetivo es comprender las apreciaciones sobre la funcionalidad familiar antes, durante y después de la desmovilización, en personas desmovilizadas que pertenecieron a grupos armados insurgentes. Se trabajó con tres entrevistas a profundidad orientadas hacia la percepción de la familia en tanto, adaptación, participación, recursos, afectividad, y capacidad resolutiva. Se encontró que ideas como "escapar" de la presión familiar, una mala convivencia sociofamiliar o de pareja, o ideología política discordante al común general, constituyeron elementos motivadores de ingreso al grupo armado, mientras que, los cambios en las ideas de familia permanecieron relativamente estables, con una clara tendencia a la independencia y desvinculación de sus familias de origen después de la desmovilización. En las familias desmovilizadas se vieron muy afectadas las áreas de adaptación, participación y consecución de recursos

Funcionalidad Familiar;

PALABRAS

Reintegración;

Conflicto Armado;

CLAVE

Grupos Armados Insurgentes;

Desmovilizado.

1 Reina Mosquera Melany, estudiante de VII semestre Psicología, Fundación Universitaria del Área Andina, mreina5@estudiantes.areandina.edu.co

2 Giraldo Ríos Dahiana, estudiante VII semestre Psicología, Fundación Universitaria del Área Andina, dgiraldo35@estudiantes.areandina.edu.co

3 Psicólogo. Docente investigador Fundación Universitaria del Área Andina. Docente investigador Universidad de San Buenaventura Medellín extensión Armenia. Magister en Investigación Integrativa. PhD en Pensamiento complejo. Email: jandrade20@areandina.edu.co; jose.andrade@usbmed.edu.co 


\title{
Insights on the family functioning of demobilized members of armed insurgent groups based on the Coffee Axis
}

\begin{abstract}
This is a qualitative, exploratory research whose objective is to understand the appreciations of family functionality before, during and after demobilization, in demobilized people who belonged to insurgent armed groups. We worked with three in-depth interviews oriented towards the family's perception in terms of adaptation, participation, resources, affectivity, and resolving capacity. It was found that ideas such as "escaping" from family pressure, poor socio-family or couple coexistence, or political ideology discordant to the common general, were motivating elements of entry to the armed group, while changes in family ideas they remained relatively stable, with a clear tendency towards independence and decoupling of their families of origin after demobilization. In the demobilized families, the areas of adaptation, participation and achievement of resources were very affected.
\end{abstract}

Family Functionality;

Reintegration;

KEYWORDS Armed Conflict;

Insurgent Armed Groups;

Demovilized. 


\section{INTRODUCCIÓN}

El conflicto armado colombiano tiene múltiples actores sociales que le dan forma y sentido a la violencia y el exceso de poder, en el marco de la confrontación estadoinsurgencia, relación destructiva que deja como víctimas a más de 8 millones de personas, familias, grupos, comunidades (Centro de Memoria Histórica - CMH, 2018; Portafolio, 2017b), y que afecta también a todos los ecosistemas del territorio. Las victimas individualmente y en conjunto se ven afectados en todos los niveles de su vida a razón de las derivaciones destructivas y lineales que la guerra suscita (Insuasty \& Vallejo, 2012; Molano, 2015; Morales, 2017; Pécaut, 2003; Villa, 2016). En este sentido, conviene mencionar que, los actores armados también pueden tipificarse como víctimas del mismo conflicto dado que, sobre ellos recaen códigos de lenguaje y de comportamiento, reglas militares de convivencia y en muchos aspectos, abusos y excesos de poder de quienes los lideran (Periódico El Espectador, 2016; Rueda, 2013). Estos aspectos forman parte del entramado de circunstancias que componen reticularmente el conflicto armado, y son también, elementos desde los cuales es factible construir una mirada relacional sobre la guerra, sus implicancias, derivas y emergencias.

Según lo señala Agencia para la Reincorporación y la Normalización (ARN, 2019) las personas a la fecha 60.424 personas se han reintegrado, siendo Antioquia el departamento con mayores tasas, pues representa el 20,40\% de total nacional. Uno de los escenarios afectados por la guerra es la familia, específicamente las dinámicas intrafamiliares que guardan relación, vínculo o que pueden ser detonantesmotivadoras del ingreso a un grupo armado (Schönrock, 2005). No quiere decir que la familia tome la decisión por quien elige formar parte de un grupo subversivo, sino que es notable que algunas personas comprendan el ingreso a un grupo armado como una vía rápida y efectiva de escape ante la presión intrafamiliar y social de la cual son objeto (Cardona, 2015; D. Giraldo, Reina, Suárez, \& Andrade, 2018). En el marco del conflicto armado existen familias acogidas a procesos de reintegración, de modo que el trabajo de reajuste psicosocial demanda competencias y metodologías de intervención diversas que logren poner en contexto las diferentes dinámicas que dan forma y sentido a su estado emocional; de allí que una adecuada intervención familiar se sostenga sobre la base de las emociones, sentimientos y percepciones sobre el antes, durante y después de la desmovilización, lo cual permite una óptica más amplia de las formas como personas, familias y grupos logran ajustarse a los nuevos territorios, contextos y demandas de entorno de socialización (Periódico El Espectador, 2016; Rueda, 2013; Schönrock, 2005).

En ese aspecto, comprender contextualmente las dinámicas familiares y la funcionalidad familiar, constituye una necesidad, al tiempo que un aporte al estudio de estas poblaciones y los procesos de reincorporación a la vida social y civil (Periódico El Espectador, 2016). Es importante mencionar que se denomina desmovilizado en proceso de reintegración a toda persona que ha abandonado las filas de un grupo armado insurgente, con el fin de acoplarse a la justicia especial para la paz y a las normas vigentes en el estado social democrático y de derecho (Congreso de la República de Colombia, 1993). Cabe anotar que la funcionalidad familiar es 
el resultado naciente de todas aquellas acciones que en colectivo se implementan para optimizar la calidad y expectativa de vida del grupo familiar, tomando como base las interacciones internas y externas, y todas aquellas ganancias y conflictos que se deriven de dichas interrelaciones (Satir, 1991, 2002), siendo "nutricias" o funcionales, aquellas familias que acogen y sostienen en múltiples niveles a sus integrantes, brindándoles apoyos y ajustando sus dinámicas a los diferentes problemas y tensiones propias de la interacción con otros sistemas (Minuchin, 2003).

Para el caso de las personas y familias en proceso de reintegración, se denota la interinfluencia de múltiples sistemas en interrelación que pueden encontrarse en conflicto y a la vez constituirse en fuente de insatisfacción constante-sistema social, comunitario, ambiental, político, laboral, etc.-, dada la presión que dichos sistemas -sociales, morales, económicos, políticos, legales, etc., -y que a la vez ejercen presión sobre el tipo de funcionalidad familiar vivido (Andrade \& Buitriago, 2011; Roca \& Riccardi, 2014). Cabe anotar que la no atención de la familia desmovilizada conforme a sus necesidades y demandas, puede originar una revictimización en diversos contextos, lo cual puede aumentar su vulnerabilidad biopsicosocial y también, las brechas con las instituciones, grupos y comunidades receptoras, lo cual está relacionado a su vez con la capacidad de conseguir un trabajo, generar redes de apoyo y superar las necesidades básicas insatisfechas (Portafolio, 2017a; Schönrock, 2005). Es importante mencionar que factores como, la no-integración comunitaria, el señalamiento social, la exclusión, discriminación y otros elementos asociados con la incapacidad de acceso material a bienes y servicios, pueden actuar conjuntamente para modificar su deseo y facultad-motivación para permanecer en la legalidad (Caracol radio, 2018; Cardona, 2015; Dinero, 2017), y con ello lograr un proceso de ajuste positivo al contexto social, así las cosas, sino se brindan las condiciones de posibilidad para la estabilidad de las personas desvinculadas de grupos armados, existe el peligro real de que estas regresen al grupo armado o que delincan en otros escenarios de violencia e insurgencia (ACR \& Agencia Colombiana para la Reintegración, 2017).

\section{MÉTODO}

Diseño de la investigación: esta es una investigación cualitativa abordada desde la Teoría Fundamentada la cual hace posible el alcance conceptual de la información recolectada y el desarrollo de las características de las categorías estipuladas operacionalmente.

Descripción de la muestra y de los escenarios: se trabajará con las narrativas expresas por 3 excombatientes desmovilizados en condición de reintegración a la vida civil, que se encuentran asentados en el Eje Cafetero, y residentes de espacios urbanos, de perfil único en tanto ex-integrantes de grupos armados por convicción/adherencia ideológica. La muestra asociada al estudio de caso, es de tipo no representativa, noprobabilística y con un muestreo de tipo teórico hasta saturación de categorías.

Aspectos éticos: se aplicará un consentimiento informado avalado por el Comité de 
Bioética. Se especifican en este consentimiento: el Tratamiento anónimo de los datos, el respeto y confidencialidad, el no recibir pago alguno y la posibilidad de abandonar el estudio cuando lo considere necesario y sin explicaciones.

Técnica cualitativa de elección: se aplicó una entrevista a profundidad, grabada y transcrita textualmente. Luego, las narrativas serán sometidas a la codificación y el análisis de contenido. La entrevista fue validada por expertos y en ella se exploró la funcionalidad familiar percibida, antes de ingresar al grupo armado; durante la estancia en el grupo; después de abandonar el grupo (desmovilización), y en función de las categorías: adaptación, participación, gradiente de recursos, afectividad, recursos o capacidad resolutiva.

Procedimiento: se realizó la definición del problema de investigación; selección de narrativas y escenarios de análisis; definición del marco temporal de estudio y acceso al campo; selección de estrategias metodológicas (teoría fundamentada, codificación abierta, axial, selectiva y condicionada). El diseño es de tipo emergente puesto que, busca explorar y conocer las características de las categorías además de la dinámica asumida por los conceptos en torno a las experiencias narradas por sus sujetos; declaración de aspectos éticos, recolección de la información, y análisis de las narrativas a través de matrices de contenido intertextual; finalmente la redacción del informe.

Fiabilidad y validez: la entrevista se validó a juicio de tres expertos en el tema. La validez de la información teórica se da con base en el uso de fuentes confiables y bases de datos.

\section{RESULTADOS Y DISCUSIÓN}

Adaptación antes, durante y después de la vinculación al grupo insurgente: existen en las personas desmovilizadas y reintegradas antecedentes de conflictos intrafamiliares, especialmente en la relación materna y en la relación de pareja, de tal modo que, para algunos, el ingreso al grupo armado constituyo una especie de escape ante dichas presiones y conflictos. De lo anterior se puede señalar que existen conflictos que preceden al grupo armado, pero que se solucionan levemente al ingresar a otro grupo de socialización, de modo que las personas pueden sentir que el grupo y su ideología los acoge, constituyen una especie de enganche para permanencia en la filas insurgentes (Giraldo, 2014). Así durante el tiempo que estuvieron en el grupo, para algunos fue fácil y para otros con el tiempo se fue haciendo fácil, pero en realidad, la permanencia en el grupo resulta difícil, y muchos quieren irse y salir, pero sobrevivir para optar por mejores condiciones de vida futura. A menudo la violencia interna en los grupos es notable y directa, especialmente contra la mujer, la cual es instrumentalizada, especialmente cuando fue reclutada de forma forzada (Villellas, 2010), la idea de escapar se asocia a los efectos sobre la salud mental y la calidad de vida, al tiempo que, la añoranza de formas previas de interacción social, aspectos muy poco tenidos en cuenta en los procesos de reintegración (Guerrero, 2014; Molinares, 2013). Se encontró que después de abandonar el grupo armado, los excombatientes 
lograron conformar una nueva familia y retomar la que tenían antes y, que durante su participación el grupo armado primaron sentimientos de amistad y cooperación mientras que "afuera del grupo" emergen sentimientos de soledad, individualismo y desconfianza, como eje transversal de muchas interacciones con otros.

Participación antes, durante y después de la vinculación al grupo insurgente: se encontró que algunas personas no sentían que tomaban sus propias decisiones, y es hasta el momento en que deciden integrarse al grupo que sienten que lo hacen, igualmente otros lo hicieron por arreglar su insolvencia económica, por su ideal político, curiosidad, o por reclutamiento. Asimismo, durante su participación en el grupo armado, señalan que "mandaba el silencio", y que sus actividades eran de protección los unos con los otros y, obedecer lo que los superiores ordenaban. Para algunos, la relación con sus familiares empeoró, al tiempo que aumentó también la presión de la desmovilización y reinserción. En muchos casos la inmersión en el grupo armado no soluciona los conflictos previos sino que los agranda (Giraldo et al., 2018), generando un estado de malestar permanente al que se aumentan los maltratos y señalamientos negativos, lo que incrementa el deseo de deserción o desmovilización (Cardona, 2015).

Gradiente de recursos antes y durante la vinculación al grupo insurgente: antes del ingreso al grupo insurgente, existían antecedentes de abandono y violencia social e intrafamiliar, también de trabajo desde edades tempranas, y en ocasiones de explotación infantil. Así, durante el conflicto armado las personas aprendieron valores, disciplina y fueron reideologizados, además de manejo de armas y defensa en el campo, lo cual resulta insuficiente para mantener a los combatientes de forma permanente. Muchos se retiraron de las filas a la primera oportunidad, para construir una vida nueva, aprender cosas diferentes, retornar con su familia y formar una familia propia. Finalmente, después de que las personas desmovilizadas y reintegradas salieran del conflicto armado, recibieron acompañamiento psicosocial, aprendieron a leer y escribir y también el apoyo de su familia, pareja y otros. Las amistades conservadas desde antes del ingreso al grupo son escasas. El conflicto armado suele deteriorar las alianzas entre personas, grupos y comunidades, instalando un desazón político y sociofamiliar innegable (Giraldo, 2014; Kalmanovitz, 2011; Pécaut, 1997), que suele extender la violencia hasta linealizarla buscando con ello normalizar sus efectos y legitimar su ejercicio (Andrade, 2018).

Afectividad antes, durante y después de la vinculación al grupo insurgente: antes de que las personas ingresaran al conflicto armado, la afectividad era inestable, pues la agresividad era el mediador en situaciones de conflicto, asimismo, quienes tenían una relación sentimental sufrieron violencia y conflictos permanentes. La noción de familia y seguridad se enmarca en el plano de apoyo "mis compañeros son mi familia porque me apoyan en todos los momentos dificiles". En este sentido, durante el proceso del conflicto armado muchas de las personas se sintieron aceptados y amados, cuidados por los compañeros y comenzaron a establecer relaciones familiares y relaciones de pareja, cosas que antes de ingresar al conflicto no tenían. Se encontró que después del proceso del conflicto armado las personas comenzaron a reunirse de nuevo con sus familias, muchas de estas familias recibieron a las personas con amor 
y comprensión, pero algunas familias no recibieron también a las personas ya que la afectividad que tenían por ellos ya no era la misma y también las relaciones de pareja ya no eran la mismas. A pesar de que el conflicto armado deteriora gravemente los vínculos cohesionantes, la propensión a la legitimidad del otro más allá del juicio de valor o segregación permite la re-elaboración vincular y la aceptación del otro en la legitimidad (Martín-Baró, 2000; Maturana, 1991)

Recursos o capacidad resolutiva antes, durante y después: se encontró que antes del ingreso al grupo insurgente, muchas personas buscaban resolver sus problemas a través de la religión, pensando en la muerte y otras recapacitaban en el conflicto armado, como solución. De suyo, durante el proceso, las personas no tuvieron problemas graves con compañeros, porque se ayudaban mutuamente, creando una buena relación entre ellos, hablando de los problemas para resolverlos; también recibir un sueldo los motivaba a permanecer en el grupo, pues ello significaba independencia económica. Después de abandonar el grupo las personas se preocupan más por ellos mismos que por otros, se deterioran los vínculos y la familia se convierte en una noción particular. Los recursos sobre la desmovilización aumentan, pero deben ser ganados bajo la lógica del trabajo, la cooperación y el cumplimiento de estándares laborales a los que se acoplan gradualmente (Agencia colombiana para la Reintegración - ARN, 2019; Dinero, 2017). Lo noción de familia cambia porque se siente que se requiere una reapropiación de elementos que le permitan afianzar su decisión con base en la aprobación más íntima -de pareja, hijos u otros- que social (Rueda, 2013).

\section{CONCLUSIONES}

Antes de ingresar al grupo al margen de la ley, se encontraron antecedentes de maltrato físico, psicológico y emocional, por parte de sus parejas, familias de origen y otros actores sociales; dicho maltrato se encuentra relacionado con el ingreso al grupo armado, en cuyo caso para muchos la experiencia insurgente se deriva de la necesidad de escape ante dinámicas de tensión, exclusión, señalamiento o violencia, procedentes de sus grupos de vinculación y referencia. Indican que en el grupo emerge el apoyo incondicional para cualquier tipo de labor, y en escasas ocasiones la discriminación fue teleológica, lo cual tiene relación con la estructura de género patriarcal que prevalece en las dinámicas guerrilleras; mientras que en las mujeres este tipo de percepción es contraria, pues al rol de combatiente se le suma el de servilismo y compañera sexual de compañeros y de otros líderes. Hombres y mujeres destacan que en el grupo armado se recalca principalmente la disciplina y el respeto hacia los demás, así como la ideología insurgente como vía explicativa de las acciones militares. Posterior a dejar el grupo, se evidencia en los participantes sentimientos y acciones de individualismo y desconfianza ante el entorno, de modo que prefieren mantener sus contactos antes que profundizar en ellos.

En el caso de la mujer entrevistada, la cual fue reclutada, de modo que no fue voluntaria su decisión, refiere que antes de pertenecer al grupo, tuvo problemas intrafamiliares complicados mediados a menudo por la violencia, y que ello se presentaba en muchas mujeres vinculadas a la guerrilla. Señaló que no quería estar en el grupo, sin embargo, 
se acopló a las normas impuestas e incluso salió con un hombre -como pareja-, pero señaló que fue por conveniencia. Después de abandonar el grupo, la experiencia dañina de su relación de pareja en las filas guerrilleras, dotada de tensión, presiones y señalamientos violentos, le generó un trauma que afectó psicológicamente sus áreas de desarrollo, al tiempo que capacidad de adaptación a situaciones difíciles y la integración a una relación de pareja sana. Actualmente tiene una familia y menciona que ya pudo superar relativamente todo lo que sucedió, aunque la relación con su familia directa -padres, hermanos y otros- no es tan cercana ni funcional, mientras que en su relación de pareja actual conserva elementos como la desconfianza y algunas inseguridades, aspecto que revela la presencia de una violencia lineal que se extiende a la vida familiar y social.

Las personas desmovilizadas logran identificar secuelas emocionales de difícil trámite y elaboración, de modo que persisten como traumas o segmentos de vivencias dolorosas que se incrementan con el paso del tiempo. Tampoco perciben que se reciba la ayuda idónea por parte del Estado en el tema de salud mental, y que la desmovilización no mueve correctamente los contenidos emocionales que pueden estar vinculados al trauma de guerra, en cuyo caso existe la idea de regresar al grupo armado ante la ineficiencia relativa de los sistemas de reincorporación y reintegración a la vida civil. El tema de género es crucial al momento de comprender la lógica restaurativa implicada en la desmovilización, de modo que, comprender y resignificar la estructura patriarcal puede orientar mejores intervenciones en contexto, ajustadas a los parámetros socioculturales implicados en todos los procesos restaurativos. 
REFERENCIAS 1 ACR, \& Agencia colombiana para la Reintegración. (2017). 4. Reconciliación Colombia 4: Eje Cafetero y Santaderes. Bogotá: Agencia colombiana para la reintegración. Retrieved from http://www.reintegracion.gov.co/es/sala-deprensa/Paginas/reconciliacion.aspx

2 Agencia colombiana para la Reintegración - ARN. (2019). Informe sobre personas reintegradas. Bogotá.

3 Andrade, J. A. (2018). ¿Es la violencia lineal? Linealidades y no-linealidades de la violencia. Medellín: Grupo de Investigacion y Editorial Kavilando.

4 Andrade, J. A., \& Buitriago, G. (2011). Estado de la función familiar, en población desplazada privada de la libertad en la cárcel "Peñas blancas" de Calarcá - Quindío en el último semestre de 2009. Revista Aletheia De La Corporación Universitaria Empresarial Alexander Con Humboldt, 1, 1-13. Retrieved from https://es.scribd.com/document/75496822/Estado-de-lafuncion-familiar-en-desplazados-privados-de-la-libertad-by-Jose-AlonsoAndrade-salazar

5 Caracol radio. (2018, January 18). Farc piden frenar asesinatos de desmovilizados y activistas políticos. Colprensa. Retrieved from http://caracol. com.co/emisora/2018/01/18/medellin/1516274704_340498.html

6 Cardona, L. (2015). Resignificación del sentido de vida de personas desvinculadas y desmovilizadas del conflicto y contribución de las redes de apoyo en su transición hacia la vida civil. Revista Universidad Pontificia Bolivariana., 15(1), 105-126. Retrieved from https://revistas.upb.edu.co/ index.php/informespsicologicos/article/view/5472/5074

7 Centro de Memoria Histórica - CMH. (2018, July 2). 262.197 muertos dejó el conflicto armado. Noticias, p. 15. Retrieved from http://www. centrodememoriahistorica.gov.co/noticias/noticias-cmh/262-197-muertosdejo-el-conflicto-armado

8 Congreso de la República de Colombia. Ley Na 82.3 de noviembre de 1993 (1993). Colombia.

9 Dinero, R. (2017). ¿Consiguen empleo los desmovilizados en Colombia? Pais. Retrieved from https://www.dinero.com/pais/articulo/desmovilizadoscondiciones-de-empleo-para-los-desmovilizados/245962

10 Giraldo, D., Reina, M., Suarez, C., \& Andrade, J. A. (2018). Funcionalidad familiar en actores del conflicto armado implicados en procesos de reintegración. Cuaderno de Investigaciones Semilleros Andina, 10(10), 178-193.

11 Giraldo, J. (2014). Política y guerra sin compasión, pp. 1-47. Retrieved from http://www.centrodememoriahistorica.gov.co/descargas/comisionPaz2015/ GiraldoJorge.pdf

12 Guerrero, M. H. (2014). Afectación de la familia a causa del conflicto armado interno. Studiositas, 6(1), 76-84. Retrieved from https://dialnet.unirioja.es/ servlet/articulo? codigo $=4459872$

13 Insuasty, A., \& Vallejo, Y. (2012). ¿Crear ambiente para la Paz? Kavilando, 4(1), 11-18. Retrieved from https://www.academia.edu/28990795/CCrear_ ambiente_para_la_paz

14 Kalmanovitz, S. (2011). El impacto económico del conflicto interno colombiano y un escenario de paz. Bogotá: INDEPAZ. 
15 Martín-Baró, I. (2000). Psicología social de la guerra: trauma y terapia. San Salvador: UCA Editores.

16 Maturana, H. (1991). La democracia es una obra de arte. Bogotá: Mesa redonda magisterio. Instituto para la democracia Luis Carlos Galán.

17 Molano, A. (2015). Fragmentos de la historia del conflicto armado (19202010). Contribución Al Entendimiento Del Conflicto Armado En Colombia. Comisión Del Conflicto y Sus Victimas, 58.

18 Molinares, H. (2013). Guerra irregular y constitución: Garantismo judicial de la Corte Constitucional colombiana. Cuestiones Constitucionales. https://doi. org/10.1016/S1405-9193(13)71295-2

19 Morales, L. (2017). La paz y la protección ambiental en Colombia. Propuestas para un desarrollo rural sostenible. Diálogo Interamericano. Washington, D.C: El Diálogo. Retrieved from http://static.iris.net.co/sostenibilidad/upload/ documents/envt-colombia-esp_web-res_final-for-email.pdf

20 Pécaut, D. (1997). De la violencia banalizada al terror. El caso colombiano. Controversia, (171), 9-31.

21 Pécaut, D. (2003). Violencia y politica en Colombia: Elementos de reflexión. Bogotá: Hombre nuevo editores.

22 Periódico El Espectador. (2016, May 31). Los retos de la sociedad para reinsertar un excombatiente. Colombia 2020. Retrieved from https:// colombia2020.elespectador.com/justicia/los-retos-de-la-sociedad-parareinsertar-un-excombatiente

23 Portafolio. (2017a, February 12). Más de 650 empresas del país ya emplean a excombatientes. Secciones. Retrieved from http://www.portafolio.co/ economia/empresas-que-emplean-a-excombatientes-en-colombia-503322

24 Portafolio. (2017b, April 9). 8.376.463: las víctimas del conflicto armado en Colombia. Gobierno, p. 20. Retrieved from https://www.portafolio. co/economia/gobierno/el-numero-de-victimas-del-conflicto-armado-encolombia-504833

25 Roca, M., \& Riccardi, A. (2014). Familia: Funcionalidad y efectividad como red de apoyo social en pacientes con depresión que están ingresados en el área de hospitalización de psiquiatría del hospital del IESS Dr. Teodoro Maldonado Carbo en la ciudad de Guayaquil (Spanish). Informes Psicológicos, 14(2), $81-102$.

26 Rueda, M. C. (2013). Reinserción y postconflicto: el "volver" de jóvenes ex combatientes a la vida civil. Universidad del Valle. Retrieved from http:// bibliotecadigital.univalle.edu.co/bitstream/10893/5874/1/0378641-p.pdf

27 Schönrock, P. (2005). Reinserción de ex-combatientes y cooperación internacional en Colombia. Perspectivas Internacionales / Universidad Pontificia Javeriana Cali, 2.

28 Villa, J. D. (2016). Intervenciones psicosociales en el marco de acciones de reparación a víctimas del conflicto armado colombiano. ECA- Estudios Centroaméricanos, 71(774), 81-85.

29 Villellas, M. (2010). La violencia sexual como arma de guerra. QUADERNS DE CONSTRUCCIÓ DE PAU, 15(7), 1-17. https://doi. org/10.1177/0032329206290426. 